\title{
Ziel: Das schmerzfreie Pflegeheim
}

\section{Österreichweites Studienprojekt möchte Möglichkeiten der Verbesserung aufzeigen}

Mit zunehmendem Alter nehmen Menschen Schmerz als Schicksal-gegeben hin. Abnehmende Artikulationsfähigkeit und Unwissen bei Ärzten und Pflege stehen einem effizienten Schmerzmanagement gerade bei Pflegeheimbewohnern im Weg. Eine wissenschaftliche Untersuchung in Kooperation mit einem privaten Pflegeheimbetreiber soll die Situation nun erfassen und verbessern.

Die Altersstruktur unserer Gesellschaft verschiebt sich, die Häufigkeit chronischer Krankheiten nimmt damit ständig zu und auch die Zahl jener, die an chronischen Schmerzen leiden. Während der Anteil der Betroffenen an der Gesamtbevölkerung auf etwa 25 Prozent geschätzt wird, sind es bei Bewohnern von Pflegeheimen 60 bis 80 Prozent. Das Problem: Schmerz wird einerseits als schicksalhaft betrachtet und kann bei zunehmender Demenz nicht mehr artikuliert werden. Mit einem wissenschaftlichen Forschungsprojekt möchte der private Pflegeheimbetreiber SeneCura nun gemeinsam mit dem Institut für Pflegewissenschaften der Paracelsus Medizinischen Universität (PMU) in Salzburg die Aufmerksamkeit auf den Schmerz in Pflegeheimen lenken und die Situation damit verbessern. Denn, so Univ.-Prof. Dr. Jürgen Osterbrink von der PMU bei der Präsentation des Projektes „Schmerzfreies Pflegeheim“ Mitte Oktober in Wien, „die Behandlung des akuten Schmerzes bedeutet die Prävention der Chronifizierung."

„Schmerz rührt an einer der Urängste des Menschen “, stellte Rudolf Öhlinger, Geschäftsführer von SeneCura, fest, , ,die meisten Menschen möchten alt werden, aber viele haben Angst vor den Schmerzen im Alter." Schmerz im Pflegeheim wird 2012 Jahresthema der privaten Institution sein. Ziel ist ein ganzheitlicher Ansatz der Schmerzreduktion.

Schmerz beeinflusst den Menschen auf verschiedensten Ebenen, die physische steht zunächst im Vordergrund: „Wenn es dunkel wird, wird der Schmerz laut", beschreibt Osterbrink, Vorstand des Instituts für Pflegewissenschaft an der PMU, die Gewichtung der Wahrnehmung: Ein- und Durchschlafschwierigkeiten führen zu Kraftlosigkeit, zu

einer Verschiebung des TagNacht-Rhythmus und zu Bettlägerigkeit, die wiederum einen drastischen Abbau der Muskelmasse zur Folge hat. Ein Teufelskreis, der sich in den psychischen, sozialen und intellektuellen Folgen fortsetzt: Schmerz begünstigt Depressionen, verändert das Rollen- und Beziehungsnetz und reduziert durch häufige Frustrationen wegen mangelnder Therapieerfolge die Motivation. Zur Schmerzsituation im Rahmen der stationären Altenhilfe liegen, so Osterbrink, derzeit keine Zahlen vor. Auch dieses Manko soll

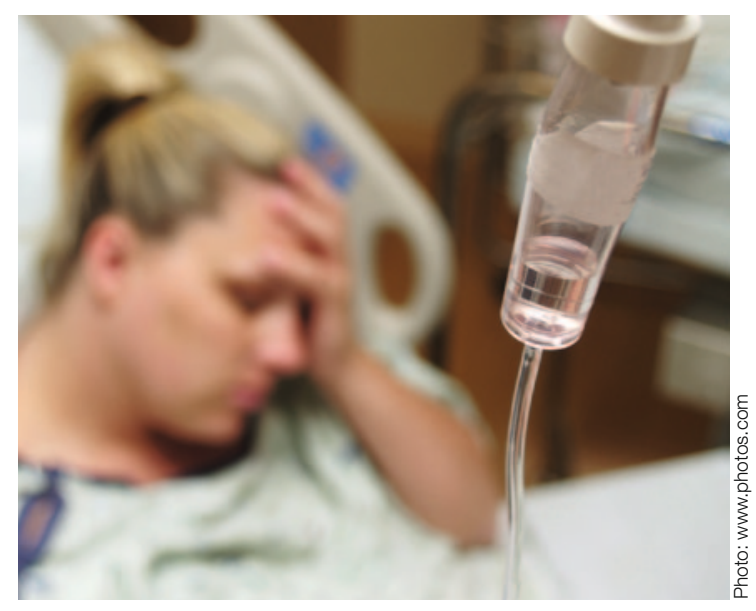

Die Relevanz von Schmerz in stationären Altenhilfeeinrichtungen ist hoch. das Projekt beseitigen. Aber: „Es besteht nicht nur viel Leid sondern auch viel Unwissen - bei Ärzten und Pflege.“

Die Relevanz von Schmerz in stationären Altenhilfeeinrichtungen ist hoch. So ergaben Studien nicht nur eine beträchtliche Häufigkeit sowohl von akuten als auch von chronischen Schmerzen sondern auch signifikant eingeschränkte kognitive Fähigkeiten (gemessen mittels Mini-Mental-Status-Test) bei betroffenen Pflegeheimbewohnern ohne Schmerztherapie. Und: „Ein signifikanter Teil von ihnen erhält keine adäquate Therapie," so Osterbrink, ,ihre Schmerzen werden oft nicht erkannt, die Schmerzstärke wird, wie eine Untersuchung von Kojer aus dem Jahr 2003 zeigt, generell unterschätzt."

\section{Ursachen für Defizite}

Die Ursachen für die Defizite im Schmerzmanagement sind vielschichtig. So gibt es etwa international keine einheitliche Systematik für die Schmerzerfassung, kein Frühwarnsystem und keine Verfahrensregeln im Tagesablauf der Pflege, Handlungsrichtlinien werden nur lückenhaft befolgt, die Einstellungen zum Schmerz sind oft mit Mythen fern der Fakten verbunden und die Kommunikation und Kooperation mit den Hausärzten könnten durchaus besser sein. Umgekehrtberichten Betroffene nur eingeschränkt über ihren Schmerz, teils aus Scham, teils ebenfalls aus Unwissenheit und teils aus
Angst, der Schmerz könnte Symptom einer bedrohlichen Erkrankung sein, mit der sie die Angehörigen nicht belasten möchten.

Ziel des Projekts ist nun die Entwicklung eines maßgeschneiderten Schmerztherapiekonzepts für die beteiligten Häuser der SeneCura. Zwölf Standorte wurden dafür Österreich-weit ausgewählt. Bis zum Jahr 2012 wird das Projekt laufen, die Erstevaluation wird im kommenden Jahr abgeschlossen sein. Mit der Reduktion der Schmerzen soll die Lebensqualität der Betroffenen erhöht, damit aber auch die Kommunikation und Zufriedenheit für die Pflege verbessert und schließlich die Wirksamkeit der eingesetzten Mittel besser genützt werden. Wenn Schmerz zu Bettlägerigkeit führt, hat dies chronische Wunden mit einem erhöhten Infektionsrisiko und damit eine intensivierte - und auch kostenintensivere - Betreuung zur Folge und schmerzbedingte Krankenhausaufenthalte wären, so Osterbrink, fast vollständig zu vermeiden. Schließlich erwartet man sich vom Projekt „Schmerzfreies Pflegeheim“ auch grundlegende wichtige Erkenntnisse $\mathrm{zu}$ Schmerz bei Bewohnern in der stationären Altenpflege und zum Schmerzmanagement bei Betagten, Hochbetagten und Dementen ebenso wie eine Verbesserung der Kommunikation und des Informationsaustauschs.

Quelle: Pressekonferenz SeneCura und Institut für Pflegewissenschaft der PMU, Salzburg 